\title{
Local Symmetry and Phason Fluctuations of an Ideal Al-Ni-Co Quasicrystal Studied by Atomic-resolution HAADF-STEM
}

\author{
Eiji Abe $^{*, * *}$ S. J. Pennycook ${ }^{*}$ and A. P. Tsai ${ }^{* *}$ \\ * Oak Ridge National Laboratory, Solid State Division, P.O. Box 2008, Oak Ridge, TN37831-6031 \\ USA \\ National Institute for Materials Science, Aperiodic Solids Research Team, 1-2-1, Sengen, \\ Tsukuba 305-0047, Japan
}

Quasicrystals are solids with symmetries forbidden to ordinary crystals. The central issues have been to locate atoms in such a non-periodic, complicated structure. The decagonal quasicrystals are built up by a periodic stacking of quasiperiodic planes (a two-dimensional quasicrystal), so that atomic-resolution imaging along the tenfold symmetry axis provides a direct interpretation of the quasiperiodic atomic structure.

An important key structural feature recently raised concerns local symmetry of quasicrystals [1-4], namely, the true symmetry of their basic building block - atomic cluster. In the case of decagonal quasicrystals, the cluster had been believed to posses a tenfold symmetry axis. The $\mathrm{Al}_{72} \mathrm{Ni}_{20} \mathrm{Co}_{8}$ is one of the best quasicrystalline compounds with nearly-perfect quasiperiodic atomic order close to its ideal stoichiometry. Atomic-resolution phase-contrast and Z-contrast images are shown in Figs. 1a and $\mathrm{b}$, in which some decagonal clusters with a diameter of about $2 \mathrm{~nm}$ are outlined to guide the eye. Viewing carefully the cluster interiors, one notices a striking feature that the contrasts appear to break tenfold symmetry at the core of the clusters [2-4]; the triangle of brightest spots representing $\mathrm{Ni}$ or $\mathrm{Co}$ is intuitive in the Z-contrast image, and the triangle modulations in the phase contrast are found to be mainly due to slight shift $(\sim 0.95 \AA)$ of $\mathrm{Al}$ atoms from the tenfold symmetry positions. The overall quasiperiodic structure can be interpreted according to the quasi-unit-cell picture [2,3] in which the symmetry-breaking decagonal clusters are allowed to overlap with their neighbors according to well-defined rules.

Originating from its high-symmetry, a unique elastic degree of freedom - termed phasons - can be defined specific to quasicrystals. This extra degree of freedom may cause a local anomaly of the Debye-Waller (DW) factor at the specified atomic sites. Here we employ the HAADF-STEM to map directly the change in thermal diffuse scattering (TDS) intensity distribution in the quasicrystal, through an in-situ high-temperature observation of the decagonal $\mathrm{Al}_{72} \mathrm{Ni}_{20} \mathrm{Co}_{8}$. We find that, at $1100 \mathrm{~K}$, a local anomaly of thermal vibrations monitored by anomalous HAADF-contrasts occurs at the core of some decagonal clusters (FIG.2), the long-range distribution of which is not random but quasiperiodically well-correlated on a length scale of 2nm [5]. Quite interestingly, we are able to explain this feature by assuming an anomalous temperature (DW) factor for the Al atoms those sit at the phason-related sites defined within the framework of hyperspace crystallography [5]. We check the DW factor effect on HAADF image contrast by changing the angular range of the detector. The origin of some phason-related structural disorders will also be discussed.

\section{References}

[1] E. Abe, T. J. Sato and A. P. Tsai, Phys. Rev. Lett. 82 (1999) 5270.

[2] P.J. Steinhardt, H.-C. Jeong, K. Saitoh, M. Tanaka, E. Abe and A.P. Tsai, Nature 396 (1998) 55.

[3] E. Abe, K. Saitoh K, H. Takakura, A. P. Tsai, P. J. Steinhardt and H.-C. Jeong, Phys. Rev. Lett. 84 (2000) 4609.

[4] Y. Yan and S. J. Pennycook, Phys. Rev. Lett. 86 (2001) 1542.

[5] E. Abe et al (2002) submitted. 

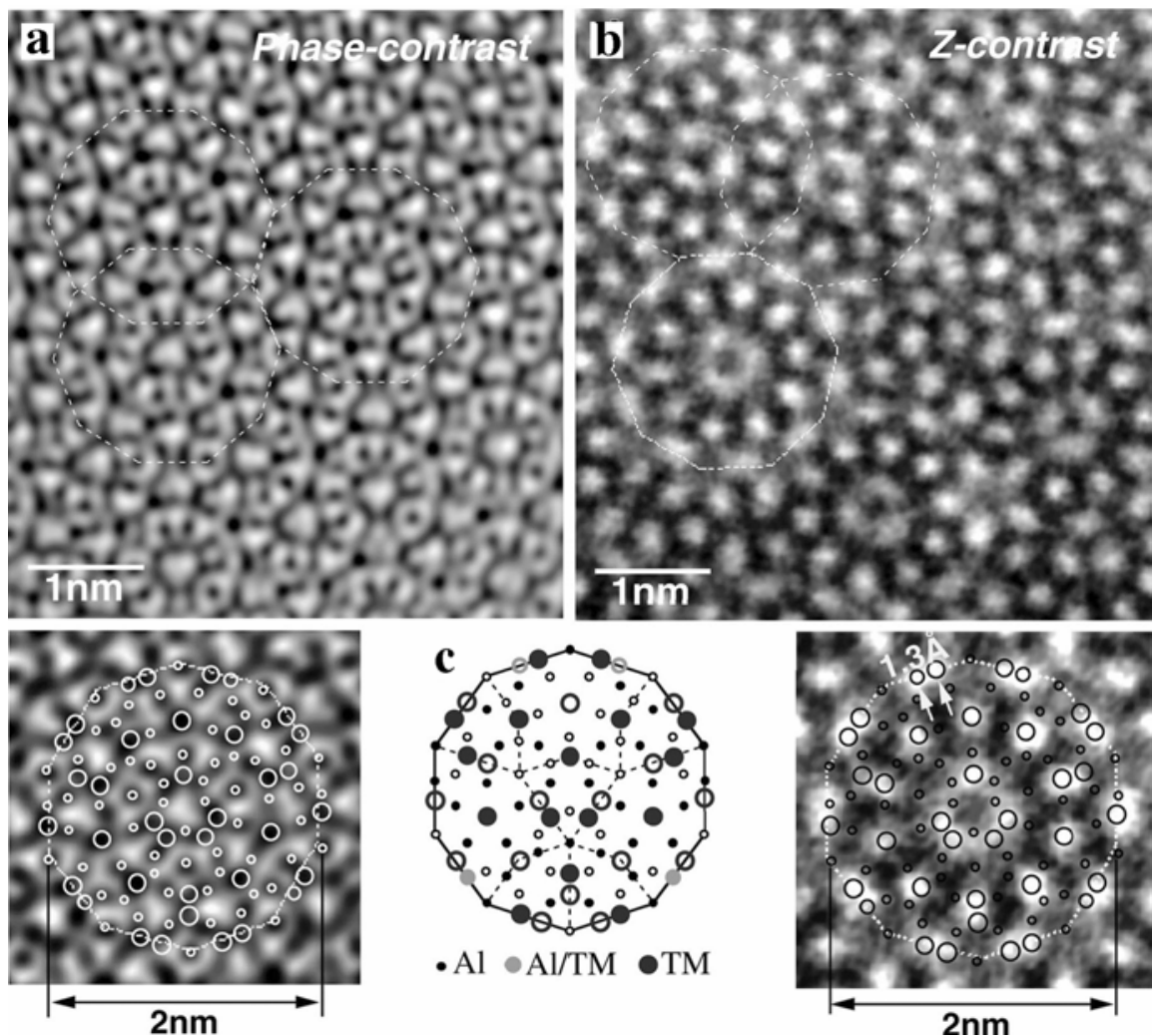

FIG. 1. Atomic-resolution (a) phase-contrast (JEM-4000EX with $\mathrm{Cs}=1.0 \mathrm{~mm}$ ) and (b) Z-contrast (JEM-2010F with $\mathrm{Cs}=0.5 \mathrm{~mm}$ ) images. (c) Atomic model of the $\mathrm{Al}_{72} \mathrm{Ni}_{20} \mathrm{Co}_{8}$, where all possible atomic positions derived from the three variations [3] of the cluster are shown. TM denotes the transition metals; Ni or Co.

High-temperature in-situ HAADF-STEM observation

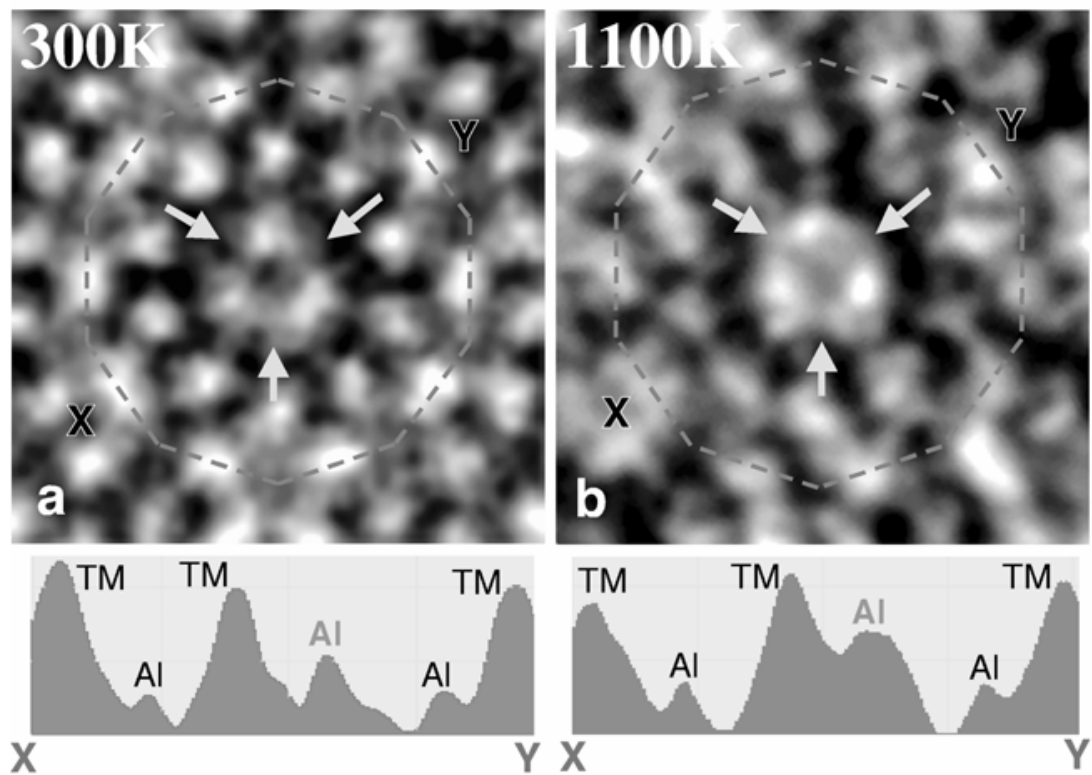

FIG. 2. HAADF images and intensity profiles across a $2 \mathrm{~nm}$-cluster at (a) $300 \mathrm{~K}$ and (b) $1100 \mathrm{~K}$. Significant contrast enhancement can be seen at the core of the cluster at $1100 \mathrm{~K}$, as indicated by arrows. This temperature-dependent contrast change is fairly well explained by an anomalous Debye-Waller factor for the $\mathrm{Al}$ atoms, related to phason fluctuations. 